\title{
Synthese und Strukturen von Bis(amino)germa- und -stanna-Chalkogeniden
}

\author{
M. Veith*, M. Nötzel, L. Stahl und V. Huch \\ Saarbrücken, Institut für Anorganischc Chemie der Universität \\ Bẹi der Redaktion eingegangen am 15. Dezember 1993.
}

Professor Wilhelm Preetz zum 60. Geburtstage gewidmet

\begin{abstract}
Inhaltsübersicht. Das cyclische Bis(amino)germylen 1 sowie das -stannylen 2 reagieren mit elementarem S, Se oder Te zu Oxidationsprodukten der allgemeinen Formel

$\mathrm{Me}_{2} \mathrm{Si}(\mathrm{NtBu})_{2} \mathrm{MEl}_{2} \mathrm{M}(\mathrm{NtBu})_{2} \mathrm{SiMe}_{2}(\mathrm{M}-\mathrm{Ge}, \mathrm{El}=\mathrm{S}(\boldsymbol{A}), \mathrm{El}-$ Se (5), $\mathrm{El}=\mathrm{Te}(\mathbf{6}) ; \mathrm{M}=\mathrm{Sn}, \mathrm{El}=\mathrm{Se}(\mathbf{9}), \mathrm{El}=\mathrm{Te}(\mathbf{1 0})$ ). Nach Röntgenstrukturanalysen $(4,5,6,9,10)$ bestehen alle Verbindungen aus drei spirocyclisch verbundenen Vierringen $\mathrm{SiN}_{2} \mathrm{M}(2 \times)$ und $\mathrm{MEl}_{2} \mathrm{M}$, die weitgehend orthogonal zueinander stehen. Die Germanium- und Zinnatome sind folglich verzerrt tetraedrisch koordiniert, während dic Chalkogenatome jeweils zwei Nachbaratome unter spityen Winkel besitzen. Setzt man 1 mit Trimethylamin-N-oxid um, so wird der Sauerstoff
\end{abstract}

an das Germanium übergeben unter Bildung von $\left[\mathrm{Mc}_{2} \mathrm{Si}(\mathrm{Nil3u})_{2} \mathrm{GeO}_{3}\right.$ (3). Im Gegensatz zu den anderen Verbindungen kann 3 formal als Trimeres aufgefaßt werden: an einem zentralen weitgehend planaren $\mathrm{Ge}_{3} \mathrm{O}_{3}$-Sechsring sind spirocyclisch an den Germaniumatomen jeweils drei $\mathrm{GeN}_{2} \mathrm{Si}$ Vicrringe geknüpft (Röntgenst.rukturanalyse von 3 ). In den zentralcn Vierringen von $4,5,6,9$ und 10 bestehen keine transanularen Wechselwirkungen zwischen den Chalkogenatomen, obwohl diesc cinen kurzen Abstand untereinander aufweisen. Die gemittclten $\mathrm{M}-\mathrm{El}$ Abstände betragen: Gc-O 1,762(5), $\mathrm{Ge}-\mathrm{S} 2,226(3)$, Ge-Sc 2,363(3), Ge-Te 2,592(5), $\mathrm{Sn} \rightarrow \mathrm{Se} 2,536(3), \mathrm{Sn}-\mathrm{Te} 2,741(3) \hat{A}$.

\section{Synthesis and Structures of Bis(amino)germa and -stanna Chalcogenides}

\begin{abstract}
The cyclic bis(amino)germylene 1 and the -stannylene 2 react with elemental $S, S e$ and Te to yield oxydation products of the general formula $\mathrm{Me}_{2} \mathrm{Si}(\mathrm{NtBu})_{2} \mathrm{MFl}_{2} \mathrm{M}(\mathrm{NtBu})_{2} \mathrm{SiMe}_{2}$ (M $-\mathrm{Ge}, \mathrm{El}=\mathrm{S}$ (4), $\mathrm{El}=\mathrm{Se}$ (5), $\mathrm{El}=\mathrm{Te}$ (6); $\mathrm{M}=\mathrm{Sn}$, $\mathrm{E} 1=\mathrm{Se}(\mathbf{9}), \mathrm{El}=\mathrm{Te}(\mathbf{1 0}))$. As may be deduced from $\mathrm{X}$-ray structures $(4,5,6,9,10)$ all compounds show similar central skeletons: the three spirocyclicly connected four-membered rings $\mathrm{SiN}_{2} \mathrm{M}(2 \times)$ and $\mathrm{MEI}_{2} \mathrm{M}$ are oricnted in an orthogonal way to oneanothcr. The germanium and the tin atoms thus are in a distorted tetrahedral coordination while the chalcogen atoms only have two neighbours in acute angles. If 1 is allowed to reacl with trimethylamine- $\mathrm{N}$-oxide, the oxygen is transferred to germanium and $\left[\mathrm{Me}_{2} \mathrm{Si}(\mathrm{NtBu})_{2} \mathrm{GeO}\right]_{3}(3)$ is formed. Contrarily to the other compounds 3 can be described as a trimer. There is a central almost planar $\mathrm{Ge}_{3} \mathrm{O}_{3}$ six-membered ring, the
\end{abstract}

germanium atoms serving as spiro-cyclic centres to three $\mathrm{GcN}_{2} \mathrm{Si}$ four-membered rings (X-ray struclure of 3 ). In the central four-membered rings of $4,5,6,9$ and 10 no transanular bonding between the chalcogen atoms have to be considered although these atoms have small distances to oneanother. The mean M-FI distances have been found to be: $\mathrm{Ge}-\mathrm{O} 1.762(5)$, Ge-S 2.226(3), Ge-Se 2.363(3), Ge-Te 2.592(5), Sn-Se 2.536(3), Sn-Te 2.741(3) $\AA$.

Keywords: 1,3-Di-tert-butyl-2,2-dimethyl-4,4-dichalcogen-1,3, 2,4-diazasilagermetidines and -stannetidines; digerma- and distanna- dichalcogencyclobutanes; oxydation of germylenes and stannylenes by chalcogens; reaction of a bis(amino)germylenc with aminc-N-oxide; $\mathrm{X}$-ray structure analyses.
Germylene, Stannylene und auch Plumbylene der allgemeinen Formel $\mathrm{MR}_{2}(\mathrm{M}=\mathrm{Ge}, \mathrm{Sn}, \mathrm{Pb} ; \mathrm{R}=$ organyl bzw. amin), die auch als carbenhomologe Verbindungen bezeichnet werden, sind seit geraumer Zeit präparativ zugänglich [1-9]. Das chemische Verhalten dieser Verbindungen wurde in den letzten 20 Jahren sehr ausführlich untersucht [vgl. Ref. [10] und die darin zitierte Literatur]. Um so erstaunlicher ist es, daß erst in jüngerer Zeit Oxidationen der Germylene und Stannylene mit den Elementen der VI. Hauptgruppe durchgeführt wurden.

So führt die Umsetzung des Stannylens $\mathrm{Sn}\left(\mathrm{NR}_{2}\right)_{2}$ $\mathrm{R}=\mathrm{SiMe}_{3}[1]$ mit elementarem Sauerstoff zu einer Ver- 
bindung, in der zwei Stannylencinheiten durch zwei Peroxogruppen verbrückt sind: [Sn( $\left.\left(\mathrm{NR}_{2}\right)_{2}\left(\mu \mathrm{O}_{2}\right)\right]_{2}$ [11]. Oxidiert man das Stannylen $\mathrm{SnR}_{2}\left[\mathrm{R}=\mathrm{CH}\left(\mathrm{SiMe}_{3}\right)_{2}\right]$ [8] mit $\mathrm{Me}_{3} \mathrm{NO}$, das formal Sauerstoff atomar abgeben kann, so erhält man als Produkt ein Distannadioxocyclobutan $\left[\left(\mathrm{SnR}_{2}\right)(\mu \mathrm{O})\right]_{2}$ [12], das erstmals einen unkoordinierten $\mathrm{Sn}_{2} \mathrm{O}_{2}$-Vierring enthält. Frühere Versuche, das zyklische Stannylen 2 [9] mit molekularem Sauerstoff zu oxidieren, führten hingegen zu einer Verbindung, in der ein $\mathrm{Sn}_{2} \mathrm{O}_{2}$-Vierring von einem weiteren Reaktionsprodukt des Stannylens 2 koordiniert wird:

$\left[\left(\mathrm{Sn}\left(\mathrm{N}^{\prime} \mathrm{Bu}\right)_{2} \mathrm{SiMe}_{2}\right)(\mu \mathrm{O})\right]_{2} \cdot\left[\mathrm{OSn}_{2}(\mathrm{NtBu})_{2} \mathrm{SiMc}_{2}\right]_{2}[13]$.

Die Umsetzung des Germylens $\mathrm{Ge}\left(\mathrm{NR}_{2}\right)_{2} \quad[R=$ $\left(\mathrm{SiMe}_{3}\right)$ ] [3] mit molekularem Sauerstoff liefert als Produkt cin Germoxan $\left[\left(\mathrm{GeNR}_{2}\right)(\mu \mathrm{O})\right]_{2}$ [14]. Ein ähnliches Digermadioxocyclobutan wurde 1989 von Masamune ct al. durch die Reaktion eines Germens $\left[R_{2} \mathrm{Ge}=\mathrm{GeR}_{2}\right]$ ( $\mathrm{R}=$ 2,6-diethylphenyl, resp 2,6-diisopropylphenyl) mit molekularem Sauerstoff erhalten und auch röntgenographisch charakterisiert [15].

Die schweren Homologen des Sauerstoffs, die Elemente Schwefel, Selen und auch Tellur reagicren ebenfalls mit Germylenen und Stannylenen. Auch hierbei werden Verbindungen erhalten, deren gemeinsames Strukturmerkmal ein Vierring darstellt, der alternierend von Elementen der IV. und VI. Hauptgruppe gebildet wird: $\left[\left(\mathrm{SnR}_{2}\right)(\mu \mathrm{S})\right]_{2} \mathrm{R}=\left[\mathrm{N}\left(\mathrm{SiMe}_{3}\right)_{2}\right]$ [16] und

$\mathrm{R}_{2}=\left[(\mathrm{NtBu})_{2}\left(\mathrm{SiMe}_{2}\right)\right]$ [17]. Läßt man elementaren Schwefel, Selen, Tellur auf ein Stannen $\left(\mathrm{SnR}_{2}\right)_{2}$ einwirken, so erhält man Verbindungen, die einen $\mathrm{Sn}_{2} \mathrm{El}_{2}$-Vierring aufweisen, $\mathrm{R}=\mathrm{tBu}, \mathrm{El}=\mathrm{S}, \mathrm{Sc}$, Te [18]. Die Umsetzung von intermediär erhaltenem Dimethylstannylen mit elementarem Tellur, führt dagegen zu einer Verbindung mit gefaltetem $\mathrm{Sn}_{3} \mathrm{Te}_{3}$-Sechsring [19].

Intramolekular basenstabilisierte Germylene und Stannylene können unter Umständen anders reagieren: die Oxidation des basenstabilisierten Germylens $\mathrm{Ge}(\mathrm{NtBu})_{4}(\mathrm{SiMe})_{2}$ durch molekularen Sauerstoff führt (ähnlich wie oben beschrieben) zu einer Verbindung mit $\mathrm{Ge}_{2} \mathrm{O}_{2}$-Vierring, die Umsetzung mit Schwefel dagegen liefert ein Produkt mit Ge-S-Doppelbindung [20]. Unsere Ergebnisse bei der Reaktion der cyclischen Amine $\mathrm{Me}_{2} \mathrm{Si}(\mathrm{NtBu})_{2} \mathrm{Ge}$ (1) und $\mathrm{Me}_{2} \mathrm{Si}(\mathrm{NtBu})_{2} \mathrm{Sn}$ (2) mit den Chalcogenen sind (soweit noch nicht geschehen) im folgenden beschrieben. Uns interessierte insbesondere die strukturelie Charakterisierung der Reaktionsprodukte, um cine Einordnung in die bereits bekannten Strukturprinzipien vornehmen zu können.

Umsetzungen des Bis(amino)germylens 1 und Bis(amino)stannylens 2 mit elementarem Schwefel, Selen und Tellur, sowie die Reaktion des Germylens 1 mit $\mathrm{Me}_{3} \mathrm{NO}$

Die in Benzol oder Toluol gelösten Edukte 1 [4] und 2 [9] reagieren mit äquimolaren Mengen der Elemente Schwefel, Selen und Tellur zu Verbindungen der allgemeinen Formel $\left[\mathrm{Me}_{2} \mathrm{Si}(\mathrm{NtBu})_{2} \mathrm{M}(\mu \mathrm{El})\right]_{2}$ (Gl. (1)).
$2 \mathrm{Me}_{2} \mathrm{Si}(\mathrm{NtBu})_{2} \mathrm{M}+2 \mathrm{EI} \rightarrow\left[\mathrm{Mc}_{2} \mathrm{Si}\left(\mathrm{N}(\mathrm{Bu})_{2} \mathrm{M}(\mu \mathrm{EI})\right]_{2}\right.$

$\mathrm{M}=\mathrm{Ge}(\mathbf{1}) \quad \mathrm{M}-\mathrm{Ge}, \mathrm{El}=\mathrm{S}(\mathbf{4})$

$\mathrm{M}=\mathrm{Sn}(\mathbf{2}) \quad \mathrm{M}=\mathrm{Ge}, \mathrm{El}-\mathrm{Se}(\mathbf{5})$

$\mathrm{M}=\mathrm{Ge}, \mathrm{El}=\mathrm{Te}(\mathbf{6})$

$\mathrm{M}=\mathrm{Sn}, \mathrm{El}=\mathrm{O}\left(7 \cdot \mathrm{Me}_{2} \mathrm{Si}(\mathrm{NtBu})_{2} \mathrm{Sn}_{2} \mathrm{O}\right)[13]$

$M-S n, E l=S(8)[17]$

$\mathrm{M}-\mathrm{Sn}, \mathrm{El}=\mathrm{Sc}(\mathbf{9})$

$\mathrm{M}=\mathrm{Sn}, \mathrm{EI}=\mathrm{Te}(\mathbf{1 0})$

Die Ausgangsverbindung 1 setzt sich mit Schwefel, in Toluol als Lösemittel, selbst bei $-72^{\circ} \mathrm{C}$ innerhalb weniger Stunden quantitativ zu Verbindung $4 \mathrm{um}$. Die Umsetzungen von 1 und 2 mit elementarem Selen und Tellur werden in Toluol durchgeführt. Der Zeitaufwand für diesc Umsetzungen ist beträchtlich ( 3 Tage bei $80^{\circ} \mathrm{C}$ ), da 1 und $\mathbf{2}$ in heterogener Phase mit festem Selen oder Tellur reagieren müssen.

Dic Bildung von $\mathbf{4}$ deutet sich ähnlich wie bei 8 [17] durch das Ausfallen eines farblosen, mikrokristallinen Niederschlages der Verbindung 4 an. Die anderen Umsetzungsprodukte von 1 und 2 mit Selen und Tellur sind jeweils in Form rotgefärbter Kristalle aus der Reaktionslösung isolicrbar. Bemerkenswert ist die geringer werdende Löslichkeit von 4, 5, 6 bzw. 8,9 und 10 mit zunehmender Ordnungszahl des $\mu$-Brückenatomes El (zur Bczeichnung vgl. Gl. (1)). Die Formulierung der Produkte in Gl. (1) als Dimere der Formel $\left[\mathrm{Mc}_{2} \mathrm{Si}(\mathrm{NtBu})_{2} \mathrm{M}-\mathrm{El}\right]_{2}$ folgt aus den Massenspektren. Wird 1 mit $\mathrm{Me}_{3} \mathrm{NO}$ bei $-70^{\circ} \mathrm{C}$ in Toluol umgeset 7. , so wird eine Verbindung erhalten, die formal als Trimeres eines Bis(amino)germaniumoxids aufgefaßt werden kann, wie sich sowohl aus der kryoskopisch bestimmten Molmasse als auch aus dem Massenspektrum ergibt (Gl. (2)).

$3 \mathrm{Me}_{2} \mathrm{Si}(\mathrm{NtBu})_{3} \mathrm{Ge}+3 \mathrm{Me}_{3} \mathrm{NO} \rightarrow\left[\mathrm{Me}_{2} \mathrm{Si}\left(\mathrm{N}(\mathrm{Bu})_{2} \mathrm{GeO}\right]_{3}+3 \mathrm{Me}_{3} \mathrm{~N}\right.$

Verbindung 3 läßt sich in Form farbloser prismenartiger Kristalle aus der Reaktionslösung durch Abkühlen isolieren. Allen Verbindungen gemcinsam ist ein auffälliger Tieffeldshift der ${ }^{t} \mathrm{H}$-Resonanzen der tert-Butylgruppen an den Stickstoffatomen und gleichzeitig ein Hochfeldshift der 'H-Resonanzen der Si-Mc-Gruppen im Vergleich zu den Resonanzen der Ausgangsverbindungen 1 und 2, rel. zu TMS als internem Standard (Tab. 1 und 2). Auch dic ${ }^{13} \mathrm{C}-,{ }^{15} \mathrm{~N}-,{ }^{29} \mathrm{Si}$-Spektren zeigen charakteristische Trends in den chemischen Verschiebungen in Abhän-

Tabelle 1 'H-Resonanzen von 1 und seinen Umsetzungsprodukten (ppm rel. zu TMS)

\begin{tabular}{lllll}
\hline & & SiMe & tBu & \\
\hline $\mathrm{El}=\mathrm{O}$ & $(\mathbf{1})$ & 0,32 & 1,20 & {$[4]$} \\
$\mathrm{El}=\mathrm{S}$ & $(\mathbf{4})$ & 0,34 & 1,43 & \\
$\mathrm{El}=\mathrm{Se}$ & $\mathbf{5}$ & 0,32 & 1,51 & \\
$\mathrm{El}=\mathrm{Fe}$ & $\mathbf{( 6 )}$ & 0,29 & 1,53 & \\
\hline
\end{tabular}


Tabelle 2 'H-Resonanzen von 2 und seinen Umsetzungsprodukten (ppm rel. zu TMS)

\begin{tabular}{lcccc}
\hline & & SiMe & tBu & \\
\hline & $(2)$ & 0,37 & 1,23 & {$[9]$} \\
$\mathrm{E}=\mathrm{S}$ & $\mathbf{( 8 )}$ & 0,32 & 1,40 & {$[17]$} \\
$\mathrm{El}=\mathrm{Se}$ & $\mathbf{( 9 )}$ & 0,31 & 1,41 & \\
$\mathrm{E}=\mathrm{Te}$ & $\mathbf{( 1 0 )}$ & $\mathbf{0 , 3 1}$ & 1,46 & \\
\hline
\end{tabular}

gigkeit der Ordnungszahl des Chalcogens (vgl. experimenteller Teil). Aufgrund der erhaltenen Signalmuster, muß man bei allen erhaltenen Verbindungen eine symmetrische Koordination der Siliciumdiazagerma- und -stanna-Vierringe durch die Chalcogenatome annehmen, was sich auch in den Röntgenstrukturbestimmungen bestätigt.

\section{Röntgenstrukturanalysen von 3, 4, 5, 6, 9 und 10}

Es gelang, von den Verbindungen $3,4,5,6,9$ und 10 für Röntgenstrukturanalysen geeignete Einkristalle zu isolieren. Die Raumgruppen wurden über die Auslöschungsmuster der jeweiligen Datensätze bestimmt und wurden im Laufe der Verfeinerung der Strukturen überprüft. Die wichtigsten Ergebnisse der Kristalluntersuchungen und der Gang der Rechnungen sind in Tabelle 3 zusammengefaßt. Eine ausführliche Zusammenstellung aller Rechenergebnisse, experimenteller Größen und der $F_{o} / F_{c}-$ Tabellen ist hinterlegt [21].

\section{Beschreibung der Struktur von $\left.\left(\mathrm{Me}_{2} \mathrm{Si}(\mathrm{NtBu})_{2} \mathrm{Ge}\right)(\mu \mathrm{O})\right]_{3}$} (3)

Abbildung 1 enthält Verbindung 3 unter der Berücksichtigung der thermischen Bewegung der Atome (30\% der Schwingungsellipsoide). Wegen der besseren Übersichtlichkeit wurde auf eine Darstellung der Wasserstoffatome verzichtet. Die Numerierung der Atome der Germasilazanringe $\mathrm{Ge} 2$ und $\mathrm{Ge} 3$ ist analog zu dem Ring, der Ge1 enthält.

Molekül 3 hat keine höhere kristallographische Punktsymmetrie. Ein annähernd planarer Sechsring, der alternierend aus Germanium- und Sauerstoffatomen gebildet wird, stellt das zentrale Strukturelement dar. Senkrecht $\mathrm{zu}$ diesem Sechsring sind die Germasilazanringe wie Schaufeln eines Wasserrades angeordnet. Abbildung 2 zeigt die Anordnung der Silagermazanringe um den $\mathrm{Ge}_{3} \mathrm{O}_{3}$-Sechsring in vereinfachter Darstellung.

Tabelle 3 Kristalldaten und Angaben zu den Kristallstrukturbestimmungen von 3, 4, 5, 6, 9, 10

\begin{tabular}{|c|c|c|c|c|c|c|c|}
\hline & & 3 & 4 & 5 & 6 & 9 & 10 \\
\hline \multicolumn{2}{|l|}{ Formel } & $\mathrm{C}_{30} \mathrm{H}_{72} \mathrm{Ce}_{3} \mathrm{~N}_{6} \mathrm{O}_{3} \mathrm{Si}_{3}$ & $\mathrm{C}_{20} \mathrm{H}_{4 \times} \mathrm{Cie}_{2} \mathrm{~N}_{4} \mathrm{~S}_{2} \mathrm{Si}_{2}$ & $\mathrm{C}_{20} \mathrm{IT}_{48} \mathrm{Gc}_{2} \mathrm{~N}_{4} \mathrm{Se}_{2} \mathrm{Si}_{2}$ & $\mathrm{C}_{20} \mathrm{H}_{48} \mathrm{Ce}_{2} \mathrm{~N}_{4} \mathrm{Si}_{2} \mathrm{Te}_{2}$ & $\mathrm{C}_{20} \mathrm{H}_{48} \mathrm{~N}_{4} \mathrm{Se}_{2} \mathrm{Si}_{2} \mathrm{Sn}_{2}$ & $\mathrm{C}_{20} \mathrm{H}_{48} \mathrm{~N}_{4} \mathrm{Si}_{2} \mathrm{Sn}_{2} \mathrm{Te}_{2}$ \\
\hline \multicolumn{2}{|l|}{ Molmasse } & 855,01 & 610,11 & 703,9 & 801,18 & 795,06 & 893,4 \\
\hline \multicolumn{2}{|l|}{ Raumgruppe } & $\overrightarrow{P 1}$ & $P 2_{1} / \mathrm{n}$ & $\mathrm{P} 2_{1} / \mathrm{n}$ & $\mathrm{Pj}$ & $P \bar{l}$ & $\mathrm{P} 2_{1} / \mathrm{n}$ \\
\hline \multicolumn{2}{|l|}{ Gitterparameter } & $10,70(2)$ & $6,420(7)$ & $6,462(7)$ & $6,676(9)$ & $6,531(6)$ & $6,728(2)$ \\
\hline \multirow[t]{5}{*}[\AA]{] bzw. $\left[^{\circ}\right]$} & $b=$ & $13,41(2)$ & $12,540(10)$ & $12,657(12)$ & $10,56(2)$ & $10,673(7)$ & $26,425(5)$ \\
\hline & $\mathrm{c}=$ & $17,12(2)$ & $19,14(2)$ & $19,19(2)$ & $13,23(2)$ & $13,470(10)$ & $19,181(4)$ \\
\hline & $\alpha=$ & $95,97(12)$ & 90 & 90 & $109,97(13)$ & $110,38(6)$ & 90 \\
\hline & $\beta=$ & $96,94(12)$ & $97,86(9)$ & $98,15(9)$ & $94,76(13)$ & $93,55(7)$ & $91,89(3)$ \\
\hline & $y=$ & $109,91(12)$ & 90 & 90 & $107,29(11)$ & $107,28(6)$ & 90 \\
\hline \multicolumn{2}{|l|}{ Zellvolumen $\left[\AA^{3}\right]$} & $2265(6)$ & $1527(3)$ & $1553(3)$ & $820(2)$ & $825,9(11)$ & 3408,3 \\
\hline \multirow{2}{*}{\multicolumn{2}{|c|}{$\begin{array}{l}\text { Zahl d. Form. Einh. } \\
\text { Dichte } D_{x}\left[\mathrm{~g} / \mathrm{cm}^{3}\right]\end{array}$}} & 2 & 2 & 2 & 1 & 1 & 4 \\
\hline & & \multicolumn{6}{|c|}{ Max. u. min } \\
\hline \multicolumn{8}{|l|}{ Zahl der unabh. } \\
\hline Reflexe & & 5213 & 2406 & 2302 & 1696 & 2562 & 5341 \\
\hline \multicolumn{8}{|l|}{ Zahl der beob. } \\
\hline Reflexe & & 4369 & 2171 & 1979 & 1601 & 2444 & 4147 \\
\hline$\sigma$-Grenze $(F>n \sigma)$ & & $2 \sigma$ & $2 \sigma$ & $2 \sigma$ & $4 \sigma$ & $2 \sigma$ & $2 \sigma$ \\
\hline $\begin{array}{l}\text { Strukturlosung } \\
\text { Lage der H-Atome }\end{array}$ & & $\begin{array}{l}\text { direkte Methoden } \\
\text { "ideale Pos.“ }\end{array}$ & $\begin{array}{l}\text { direkte Methoden } \\
\text {,ideale Pos." }\end{array}$ & $\begin{array}{l}\text { direkte Methoden } \\
\text {,idcale Pos.“ }\end{array}$ & $\begin{array}{l}\text { direkte Methoden } \\
\text { „ideale Pos." }\end{array}$ & $\begin{array}{l}\text { direkte Methoden } \\
\text { nicht berücks. }\end{array}$ & $\begin{array}{l}\text { dirckte Methoden } \\
\text { "ideale Pos." } \mathrm{m} \text {. } \\
\mathrm{C}-\mathrm{H}=0,96 \AA\end{array}$ \\
\hline \multicolumn{2}{|l|}{ Zahl der Parameter } & 479 & 161 & 184 & 165 & 137 & 320 \\
\hline R-Werle $R$ & & 4,87 & 2,46 & 3,02 & 6,33 & 4,58 & 3,49 \\
\hline $\mathbf{R}_{\mathrm{w}}$ & & 4,48 & 2,41 & & 6,88 & 4,36 & 3,04 \\
\hline \multicolumn{2}{|l|}{ Gewichtsschema ${ }^{a}$ ) } & $\mathrm{k} 0,0001$ & 0,0001 & & 0,0001 & 0,0177 & 0,0004 \\
\hline $\begin{array}{l}\text { Restelektronen- } \\
\text { dichte }\left[\mathrm{e} \AA^{-3}\right]\end{array}$ & & 0,53 & 0,30 & 0,36 & 1,11 & 0,69 & 1,07 \\
\hline
\end{tabular}

a) $\mathrm{W}=1 /\left(\sigma_{\mathrm{r}}^{2}+\mathrm{k} \cdot \mathrm{F}^{2}\right)$ 


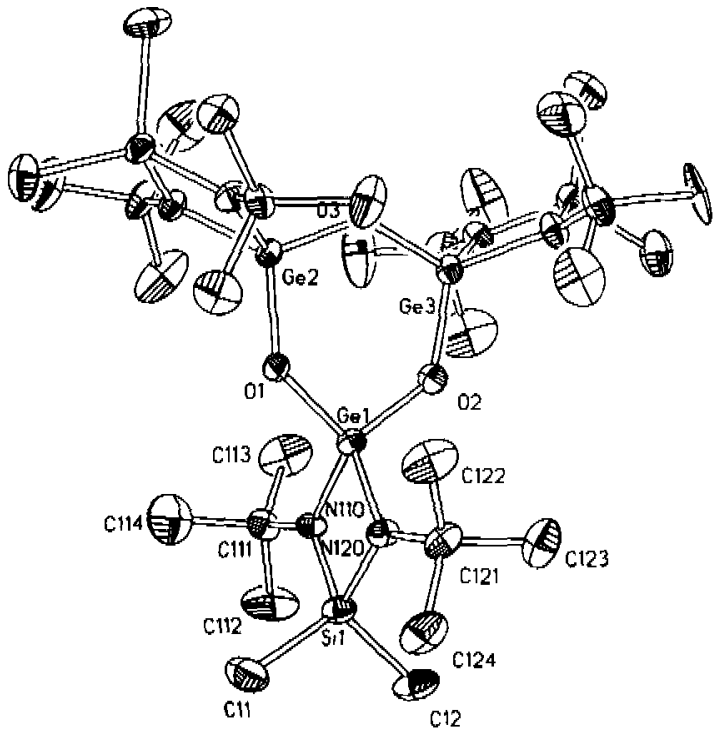

Abb. 1 Zeichnerische Wiedergabe (mit Schwingungsellipsoiden $(30 \%)$ ) der Molekülstruktur von $\left[\mathrm{Me}_{2} \mathrm{Si}(\mathrm{NtBu})_{2} \mathrm{GeO}\right]_{3}$ (3) nach Röntgenstrukturanalyse

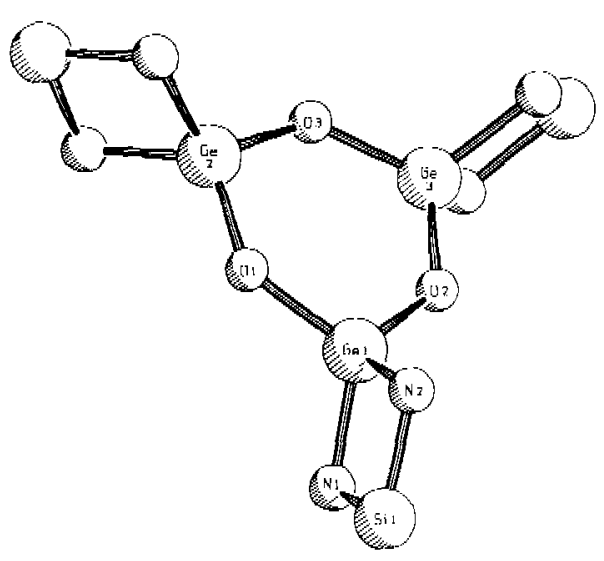

Abb. 2 Das Gerüst der spirocyclisch verknüpften Ringe in 3 (vgl. Text)

Die Germanium-Sauerstoffabstände erstrecken sich über einen Bereich von 1,743(5) $\AA$ (Ge2-O1) bis $1,773(5) \AA$ (Ge1-O2). Sie sind um $0,2 \AA$ kürzer als die Summe der kovalenten Radien von Germanium und Sauerstoff mit $1,95 \AA$. Die Bindungslängen liegen auch unter den Werten, die üblicherweise für Ge-O-Einfachbindungen gemessen werden $(1,805$ (9) $\AA-1,994(5) \AA$ [14]). Eine Mesomerie mit $\mathrm{p} \pi-\mathrm{d} \pi$ Anteilen im $\mathrm{Ge}_{3} \mathrm{O}_{3}$ Sechsring läßt sich damit nicht ausschließen. Annähernd identische Winkel $\left(134,3^{\circ} \pm 1,2^{\circ}\right)$ werden um die Sauerstoff-Atome gemessen. Die Winkel an den schwereren Germaniumatomen im $\mathrm{Ge}_{3} \mathrm{O}_{3}$-Ring sind wesentlich kleiner $\left(105^{\circ}\right)$ als an den Sauerstoffatomen. Dies könnte eine sterische Ursache (tert-Butyl-Gruppen und deren Abstoßung innerhalb des Sechsringes) haben oder einfach damit erklärt werden, daß die Sauerstoffatome bei der Anpassung im Ring von einem $\mathrm{sp}^{2}$-Hybridisierungszu- stand ausgehen (vielleicht sind auch beide Effekte zu beachten). Wie erwartet übernimmt das weichste und schwerste Atom in Gestalt des Germaniumatoms $(\mathrm{N}-\mathrm{Gc}-\mathrm{N}=82,40)$ die Hauptlast der Ringspannung in den Germasilazanringen.

\section{Strukturbeschreibungen der Verbindungen 4, 5, 6, 9 und 10}

Wie die Ergebnisse der Röntgenstrukturanalysen von 4, 5, 6, 9 und 10 zeigen, haben alle diese Verbindungen eine analoge Molekülstruktur: sie bestehen aus den drei spirozyklisch verbundenen Vierringen, $(\mathrm{MEl})_{2}$ und aus je zwei $\mathrm{SiN}_{2} \mathrm{M}$-Ringen, die weitgehend orthogonal zueinander stehcn. Als kristallographische Punktsymmetrie haben alle Moleküle außer 10, dessen Punktsymmetrie jedoch nicht viel davon abweicht, ein Inversionszentrum. In Abb. 3 ist als Beispiel für dic allesamt sehr ähnlichen Moleküle die Verbindung $\left[\mathrm{Me}_{2} \mathrm{Si}(\mathrm{NtBu})_{2} \mathrm{GeS}\right]_{2}$ (4) wiedergegeben.

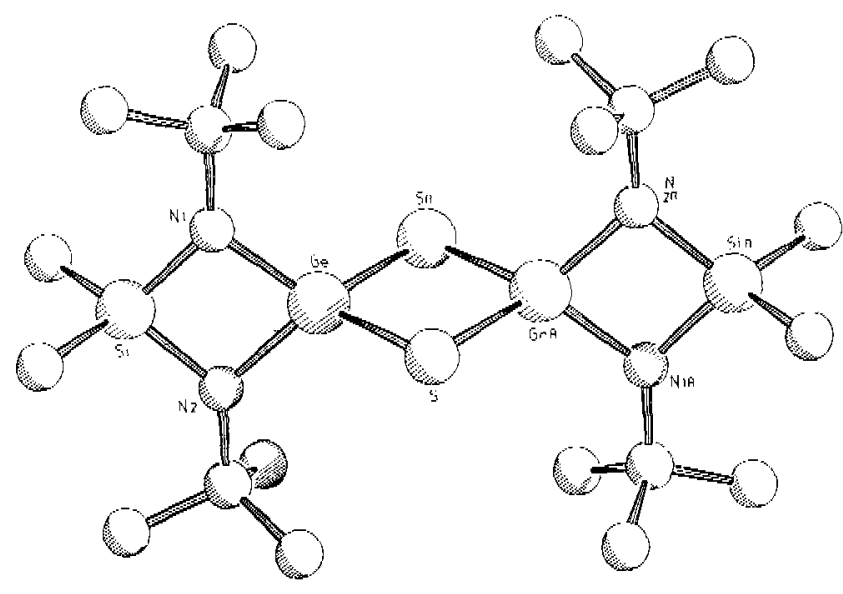

Abb. 3 Molckülstruktur von $\left[\mathrm{Me}_{2} \mathrm{Si}\left(\mathrm{N}(B u)_{2} \mathrm{GcS}_{2}\right.\right.$ (4) nach Röntgenstrukturanalyse. Die Strukturen von 5, 6, 9 und 10 sind dem hier gczcigten Molekül sehr ähnlich

Die Geometrie in den $\operatorname{SiN}_{2} \mathrm{M}$-Ringen $(\mathrm{M}=\mathrm{Ge}$ bzw. $M=S n$ ) wird offenbar durch Auswechseln der Chalcogen-Brückenatome wenig beeinflußt (vgl. Tab. 4), wobei insbesondere auffällt, daß die $\mathrm{M}-\mathrm{N}$-Bindungslänge, trotz Variation des Chalcogenatoms, weitgehend konstant bleibt.

In den zentralen $(\mathrm{MEl})_{2}$-Vierringen ändern sich die M-El-Bindungsabstände mit steigender Ordnungszahl

Tabelle 4 Bindende und nichtbindende Abstände $(\AA)$ (gemittclt) in den Ringen $\mathrm{SiN}_{2} \mathrm{M}(\mathrm{M}=\mathrm{Ge}, \mathrm{Sn})$ von 3, 4, 5, 6, 9, 10

\begin{tabular}{lllllll}
\hline Ge-O & Ge-S & Ge-Se & Ge-Te & Sn-Se & Sn-Te \\
\hline Si-M & $2,605(5)$ & $2,608(6)$ & $2,611(4)$ & $2,625(8)$ & $2,784(3)$ & $2,790(4)$ \\
N-N & $2,431(6)$ & $2,431(6)$ & $2,412(5)$ & $2,440(7)$ & $2,538(4)$ & $2,505(5)$ \\
N-M & $1,824(7)$ & $1,832(3)$ & $1,826(2)$ & $1,852(10)$ & $2,023(8)$ & $2,030(6)$ \\
N-Si & $1,728(8)$ & $1,738(3)$ & $1,731(3)$ & $1,735(14)$ & $1,741(4)$ & $1,730(3)$
\end{tabular}


Tabelle 5 Bindende und nichtbindende Abstände $(\AA)$ und Winkel $\left(^{\circ}\right)$ (gemittelt) in dem Ring $(\mathrm{MEl})_{n}(\mathrm{n}=2,3)(\mathrm{M}-\mathrm{Ge}, \mathrm{Sn}$; $\mathrm{El}=\mathrm{O}, \mathrm{S}, \mathrm{Se}, \mathrm{Te})$

\begin{tabular}{|c|c|c|c|c|c|c|}
\hline & $\mathrm{Gc}-\mathrm{O}$ & $\mathrm{Gc}-\mathrm{S}$ & $\mathrm{Ge}-\mathrm{Se}$ & $\mathrm{Ge}-\mathrm{Te}$ & $\mathrm{Sn}-\mathrm{Se}$ & $\mathrm{Sn}-\mathrm{Te}$ \\
\hline $\mathbf{M}-\mathbf{M}$ & $3,245(4)$ & $3,007(3)$ & $3,146(3)$ & $3,416(6)$ & $3,371(3)$ & $3,582(4)$ \\
\hline $\mathrm{El}-\mathrm{El}$ & $2,806(3)$ & $3,294(4)$ & $3,526(3)$ & $3,898(4)$ & $3,790(5)$ & $4,148(4)$ \\
\hline $\mathrm{M}-\mathrm{E} 1$ & $1,762(5)$ & $2,226(3)$ & $2,363(3)$ & $2,592(5)$ & $2,536(6)$ & $2,741(5)$ \\
\hline $\mathrm{El}-\mathrm{M}-\mathrm{El}$ & $105,7(1)$ & $95,2(1)$ & $96,5(1)$ & $97,5(2)$ & $96,7(1)$ & $98,4(1)$ \\
\hline $\mathrm{M}-\mathrm{El}-\mathrm{M}$ & $134,3(1)$ & $84,8(1)$ & $83,5(1)$ & $82,5(2)$ & $83,3(1)$ & $81,6(1)$ \\
\hline
\end{tabular}

bzw. Größe aller an der Ringbildung beteiligter Atome. Dies wirkt sich sowohl auf die transanularen Abstände $\mathbf{M}-\mathbf{M}$ als auch auf die $\Lambda$ bstände $\mathrm{El}-\mathrm{El}$ aus (vgl. Tab. 5).

Trảgt man den Abstand der Chalcogenatome El-El gegen den Abstand $\mathbf{M}-\mathbf{M}$ auf, so liegen alle Punkte innerhalb der Standardabweichungen auf einer Geraden (vgl, Abb. 4). Dies legt nahe, daß spezielle transanulare Wechselwirkungen zwischen den Chalcogenatomen in einem der Derivate im Vergleich zu den anderen ausgeschlossen werden können.

Um systematisch zu untersuchen, inwieweit die Größe des Chalcogens die Abmessungen in den zentralen Ringsystemen beeinflußt, wurden die Bindungslängen $\mathrm{M}-\mathrm{El}$ bzw, die transanularen Abstände El-El als Funktion des Chalcogenradius aufgetragen (Abb. 5).

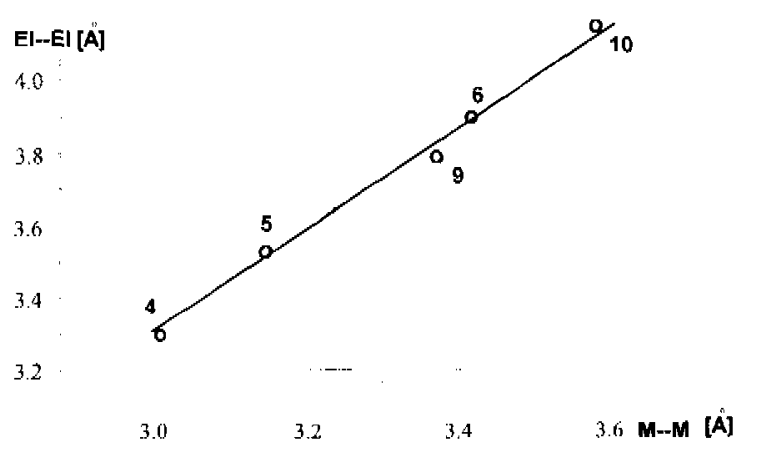

Abb. 4 Korrclation der nichtbindenden Abstände El-El/ $M-M(E l=S, S e, T e ; M=G e, S n)$ für $4,5,6,9$ und 10

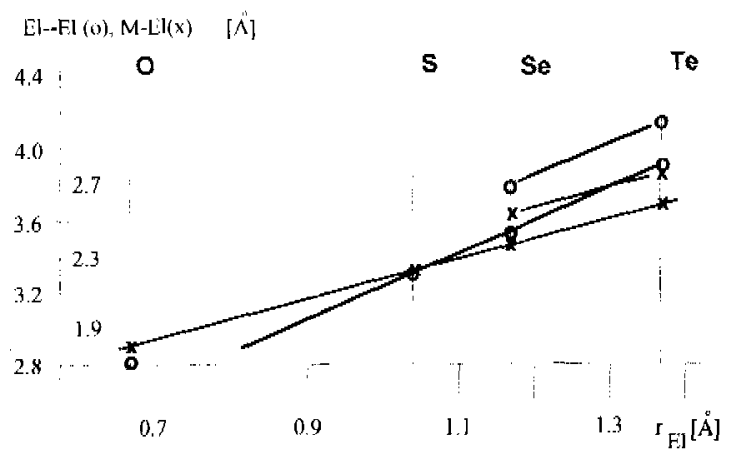

Abb. 5 Die Abhăngigkeit der El-Fl- bzw. M-El-Abstände von den Atomradien der Chalcogene für 3, 4, 5, 6, 9 und 10
Betrachtet man zunächst die Abhängigkeit der Chalcogenabstände $\mathrm{El}-\mathrm{El}$ in den Ringen $(\mathrm{GeEl})_{2}(\mathrm{El}=\mathrm{S}$, Se, $\mathrm{Te})$ und $(\mathrm{SnEl})_{2}(\mathrm{El}=\mathrm{Se}, \mathrm{Te})$ vom Radius des Brückenatoms El (vgl. Abb. 5), so erkennt man, daß diese Punke auf zwei parallelen Geraden liegen, dic durch die Atome Ge und Sn definiert sind. Dic Verbindung 3 licgt in dieser Auftragung nicht auf der Geraden: der im Sechsring gefundene $\mathrm{O} \cdots$ O-Abstand $(2,806 \AA)$ ist also größer als der nach Extrapolation der Geraden $z u$ erwartende $\mathrm{O} \cdots \mathrm{O}-\Lambda \mathrm{bstand}$ in einem hypothetischen $\mathrm{Ge}_{2} \mathrm{O}_{2}$ Vierring (etwa 2,55 $\AA$ ). In der Verbindung $\left[\left(\mathrm{Me}_{3} \mathrm{Si}\right)_{2} \mathrm{~N}\right]_{2} \mathrm{GeO}_{2} \mathrm{Ge}\left[\mathrm{N}\left(\mathrm{SiMe}_{3}\right)_{2}\right]$ beträgt det $\mathrm{O} \cdots \mathrm{O}-$ Abstand demzufolge auch 2,48(1) $\AA$ [14], ist also wesentlich kleiner als in 3. Nut auf die $\mathrm{M}_{2} \mathrm{El}_{2}$-Vierringe bezogen, legt die Auftragung des El ... El-Abstandes gegenüber dem Radius der Elemente El nahe, daß die Zunahme des El-El-Abstandes - gleichgültig, ob man die Zinnoder dic Germaniumverbindungen betrachtet - um denselben Faktor erfolgt. Der El-El-Abstand ist somit lediglich eine Funktion der Chalcogengröße. Analoge Ergebnisse werden aus der Auftragung des Abstandes $\mathrm{M}-\mathrm{El}$ gegen den Radius des Chalcogens El erhalten. Auch die Abstände $\mathrm{M}-$ El vergößern sich - unabhängig von dem Spiro-Atom - um den selben Faktor. Aus der Auftragung in Abbildung 5 ist auch zu erkennen, daß die Werte des $(\mathrm{GeO})_{3}$ Sechsringsystemes mit den Punkten der Vierringsysteme auf einer Geraden liegen. Von den Bindungslängen $\mathrm{Ge}-\mathrm{O}$ aus betrachtet, solltc sich das Sechsringsystem $\mathrm{Ge}_{3} \mathrm{O}_{3}$ nicht wesentlich von einem Vierringsystem unterscheiden (in $\left[\left(\mathrm{Me}_{3} \mathrm{Si}\right)_{2} \mathrm{~N}_{2} \mathrm{GeO}_{2} \mathrm{Ce}\right.$. $\left[\mathrm{N}\left(\mathrm{SiMe}_{3}\right)_{2}\right]_{2}$ beträgt der Ge-O-Abstand demzufolge auch 1,805(9) Å). Für die Bildung des Sechsringes sind somit außer den sterischen Wechselwirkungen der tertButyl-Liganden auch die intramolekulare Abstoßung der Sauerstoffatome verantwortlich.

Dic aufgrund des kristallographischen Inversionszentrums zueinander parallelen $\operatorname{SiN}_{2} \mathrm{M}$-Vierringe in den Verbindungen 4, 5, 6, 9 und 10 befinden sich im Festkörper nicht, wie man vermuten könnte, in eincr Ebene: sie sind ausnahmslos ,versetz1" zueinander angeordnet. Offenbar richten sich die tert-Butylgruppen an den beiden Ringen paarweise zueinander aus und beeinflussen dadurch auch die Lage der Ringe zueinander. In Abbildung 6 ist die ,Versetzung" als Abweichung der $\mathrm{M}-\mathrm{M}$ Diagonalen von den $\mathrm{Si}-\mathrm{M}$ Diagonalen (ausgezeichncte Linie) dargestellt. Die Winkel $\mathrm{M}-\mathrm{M}-\mathrm{Si}$, die 


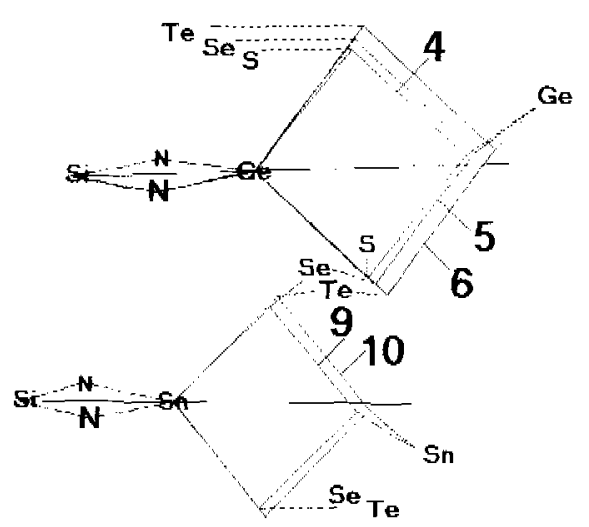

Abb. 6 Superposition der Germasilazan- und StannasilazanRinge (praktisch senkrecht zur Abbildungsebenc) und Ausrichtung bzw. Größenvergleich der $\mathrm{M}_{2} \mathrm{El}_{2}$-Ringe in 4, 5 und 6 (oben) brw. 9 und 10 (unten). Durch $\mathrm{Si}$ und $\mathrm{Ge}(\mathrm{Sn})$ ist in beiden 7eichnungen eine Gerade gerogen, um die Lage des $\mathrm{Fl}_{2} \mathrm{M}_{2^{-}}$ Ringes zum Silazan-Ring aulzuzeigen (vergleiche auch Text)

man als Maß für die Versetzung benutzen kann, betragen: $177,8^{\circ}$ (4), $177,1^{\circ}$ (5), $177,5^{\circ}$ (6), $177,0^{\circ}$ (9), $177,20^{\circ}$ (10).

\section{Zusammenfassung}

Die Carbenhomologen Bis(amino)germylen 1 und Bis(amino)stannylen 2 reagieren mit den Elementen $\mathrm{S}, \mathrm{Se}, \mathrm{Te}$ zu analogen Verbindungen. Man crhält jeweils eincn Vierring, der alternierend von Elementen der IV. und VI. Hauptgruppe gebildet witd. Dieser $\mathrm{M}_{2} \mathrm{El}_{2}$ Vicrring ändert seine Größe gleichmäßig mit der Zunahme des Metall- und des Chalcogenradius. Die Oxidation des Germylens 1 durch $\mathrm{Me}_{3} \mathrm{NO}$ unter Freisetzung von Trimethylamin führt zur Bildung eines $\mathrm{Ge}_{3} \mathrm{O}_{3}$-Sechsringes, wobei sowohl sterische als auch elektronische Gründe für diese Ausnahmestellung verantwortlich sein können.

\section{Experimenteller Teil}

Alle Umsetzungen wurden unter $\mathrm{N}_{2}$-Schutzgas in abgeschlossenen Glasapparaturen durchgeführt. Die 'H-NMR-Spektren sowie die Resonanzen der Kerne ${ }^{13} \mathrm{C},{ }^{15} \mathrm{~N},{ }^{24} \mathrm{Si}$ wurden auf einem Gerät der Firma Bruker (AC 200/Aspect 3000; $200 \mathrm{MHz}$ ) aufgenommen. Für dic Massenspcktren benutzten wir ein Finnigan MAT 90 (120 eV, CI, Reaktionsgas Methan). Die C-, H-, N-Analysen wurden vom mikroanalytischen Laboratorium Beller, Göttingen, durchgeführt. Die Datensätze zur Strukturbestimmung wurden mit einem automatischen Vierkreisdiffraktometer der Firma Siemens/Stoe, Darmstadt, Typ AED 2, erhalten und an cinem Vax-Rechner von Digital Corporation, Boston, USA, unter Zuhilfenahme der Programme SHELXS 86 [22], SHELX 76 [23], SHELXTL-PLUS [24] ausgewertet. Die graphischen Darstellungen der Molekülstrukturen wurden mit Hilfe des Programmes SCHAKAL, [25] sowie mit dem in dem Programmpaket SHELXTL-PLUS enthattenen Programm XP erstcllt.

Umsetzung von $\mathrm{Me}_{2} \mathrm{Si}(\mathrm{NtBu})_{2} \mathrm{Ge}$ mit $\mathrm{Me}_{3} \mathrm{NO}$, Darstellung von 3 $\mathrm{Zu}$ einer auf $-72^{\circ} \mathrm{C}$ gekühlten lösung von $0,158 \mathrm{~g}$ $\left(2,1 \cdot 10^{\cdot 3} \mathrm{~mol}\right) \mathrm{Me}_{3} \mathrm{NO}$ in $25 \mathrm{ml}$ Toluol werden $0,53 \mathrm{ml}$
(2 - $10{ }^{3}$ mol) des Germylens 1 gelöst in $25 \mathrm{ml}$ Toluol, innerhalb von $8 \mathrm{~h}$ zugetropft. Nach der Zugabe wird noch $10 \mathrm{~h}$ bei dieser Temperatur gerührt. Nach Erwärmung au Raumtemperatur wird das Reaktionsgemisch filtricrt und i. Vak. getrocknet. Unkristallisation aus $20 \mathrm{n}$ l Toluol hefert $0,569 \mathrm{~g}(95 \% \mathrm{~d}$. Th.) an 3 in Form farbloser, quaderförmiger Kristalle. Schmp.: $306^{\circ} \mathrm{C} ; \mathrm{C}_{30} \mathrm{H}_{72} \mathrm{Ce}_{3} \mathrm{~N}_{12} \mathrm{O}_{3} \mathrm{Si}_{3}$; Molmasse ber.: $855,01 \mathrm{~g} / \mathrm{mol}$, gef, (kryoskopisch in Benzol): $867 \mathrm{~g} / \mathrm{mol}$; C 38,3 (ber. 42,1), H 7,8 (bcr. 8,4), N 9,6 (ber. 9,8)\%. 'H-NMR: 0,33 (s, 18 H) Si--Me, $1,42(\mathrm{~s}, 54 \mathrm{H}) \mathrm{NtBu} ;{ }^{13} \mathrm{C}-\mathrm{NMR}: 5,47(\mathrm{Si}-\mathrm{C}), 35,41\left(\mathrm{C}-\mathrm{CH}_{3}\right)$, $51,39(\mathrm{C}-\mathrm{CH}),{ }^{15} \mathrm{~N}-\mathrm{NMR}:-292,78,{ }^{29} \mathrm{Si}-\mathrm{NMR}: 0,36 \mathrm{ppm} ;$ Massenspektrum: m/e (rel. Intensität) $855(7) \mathrm{M}^{+}$

Umsetzung von $\mathrm{Me}_{2} \mathrm{Si}(\mathrm{NtBu})_{2} \mathrm{Ge}$ mit elementarem Sclwefel, Darstellung der Verbindung 4

Zu ciner Lösung von $0,064 \mathrm{~g}(0,002 \mathrm{~mol})$ Schwefel in $25 \mathrm{ml}$ Toluol werden $0,5 \mathrm{ml}(0,002 \mathrm{~mol})$ des Gemylens 1 , gelöst in $25 \mathrm{ml}$ Toluol, bei $-72^{\circ} \mathrm{C}$ innerhalb $5 \mathrm{~h}$ zugetropft. Fs bildet sich schon bei der Zugabe ein farbloser, mikrokristalliner Nicderschlag. Man läßt noch weitete $12 \mathrm{~h}$ boi dicscr Temperatur rühren. Nach Beendigung der Reaktion erwärmt man auf Raumtemperatur. Das Rohprodukt wird durch Dekantieren von der überstehenden lösung getremnt. Umkristallisation aus $50 \mathrm{ml}$ Toluol liẹtert $0,567 \mathrm{~g}(93 \%)$ von 4 in form farbloser, nadelförmiger Kristalle. Schmp.: $302^{\circ} \mathrm{C} . \quad \mathrm{C}_{20} \mathrm{H}_{48} \mathrm{Ce}_{2} \mathrm{~N}_{4} \mathrm{~S}_{2} \mathrm{Si}_{2}$ (Molmasse: 610,11 ); C 39,64 (ber, 39,37), H 7,89 (7,93), N 9,12 $(9,18) \%$. 'H-NMR: $0,312(\mathrm{~s}, 12 \mathrm{H}) \mathrm{Si}-\mathrm{Me}, 1,50 \quad(\mathrm{~s}, 36 \mathrm{H})$ $\mathrm{NtBu} ; \quad{ }^{13} \mathrm{C}-\mathrm{NMR}: \quad 6,02(\mathrm{Si}-\mathrm{C}), \quad 34,21\left(\mathrm{C}-\mathrm{CH}_{3}\right), \quad 52,94$ (C- $\mathrm{CH}_{3}$ ); ${ }^{15} \mathrm{~N}-\mathrm{NMR}-295,6 ;{ }^{29} \mathrm{Si}-\mathrm{NMR} ; 4,32 ; \mathrm{MS}: \mathrm{m} / \mathrm{e}$ (rel. Intensität) $597(43,3) \mathrm{M}^{1}-\mathrm{CH}_{3}$.

\section{$\left[\mathrm{Me}_{2} \mathrm{Si}\left(\mathrm{N}(\mathrm{Bu})_{2} \mathrm{GeSe}\right]_{2}(5)\right.$}

In einem $100 \mathrm{ml}$ Zweihalskolben werden $120 \mathrm{mg}(1,52 \mathrm{mmol})$ graucs Selenpulver eingewogen. Dazl gibt man $8,0 \mathrm{ml}$ einer $0,20 \mathrm{M}$ Lösung des Germylens 1 in Toluol und verdünnt das Reaktionsgemisch mit ca. $50 \mathrm{ml}$ Toluol. Beim Erhitzen auf $60^{\circ} \mathrm{C}$ löst sich das Selenpulver schr rasch auf und man crhält eine klare, farblose Lösung. Dicse Lösung wird heil\} filtriert. Beim Erkalten bildet sich eine große Menge fciner, nadelförmiger Kristalle. Ausboute: $470 \mathrm{mg}(95,4 \%)$. Gut ausgebildetc Einkristalle crhält man, indem man eine verdünnte Toluollösung der Vcrbindung im Temperaturbereich $60-30^{\circ}$ langsam abkühlt. Die Verbindung ist erstaunlich luft- und wasserunempfindlich und in allen Kohlenwasserstoffen wcitgehend unlöslich. Schmp.: $a b$ ca. $260^{\circ} \mathrm{C}$ Zersetzung; $\mathrm{C}_{20} \mathrm{H}_{48} \mathrm{Ge}_{2} \mathrm{~N}_{4} \mathrm{Sc}_{2}$ (Molmasse: 703,9); C 34,30 (ber. 34,13$)$; H 6,73 (6,87); N 7,91 $(7,96) \%$; 'H-NMR: $0,29(\mathrm{~s}, 12 \mathrm{ll}) ; 1,53(\mathrm{~s}, 36 \mathrm{ll}) ;{ }^{13} \mathrm{C}-\mathrm{NMR}: 6,4\left(\mathrm{Si}-\mathrm{CH}_{3}, 34,0\right.$ $\left(\mathrm{C}-\mathrm{CH}_{3}\right), 53,4\left(\mathrm{C}-\mathrm{CH}_{3}\right) \mathrm{ppm}$.

\section{Umsetzung von $\mathrm{Me}_{2} \mathrm{Si}(\mathrm{NtBu})_{2} \mathrm{Gc}$ mit elementarem Tellur, Dar- stellung der Verbindung 6}

$\mathrm{Zu}$ einer Suspension von $0,254 \mathrm{~g}\left(2 \cdot 10^{-3} \mathrm{~mol}\right)$ aus fein gepulvertem Tellur in $50 \mathrm{ml}$ Toluol werden $0,5 \mathrm{~mol}\left(2 \cdot 10^{-3} \mathrm{~mol}\right)$ des Germylens 1 pipetticrt. Das Reaktionsgemisch wird $72 \mathrm{~h}$ bei $80^{\circ} \mathrm{C}$ gerührt, wobei sich dic Lösung dunkelrot verfärbı. Das Gemisch wird mit $20 \mathrm{ml}$ Toluol verdünnt und bei $70^{\circ} \mathrm{C}$ rasch filtriert. Nach Abkühlen und Einengen der tiefroten klaren Lösung erhält man $0,697 \mathrm{~g}(87 \% \mathrm{~d}$. Th.) an 6 als rote, mikrokristalline Nadcln. Schmp,; $315^{\circ} \mathrm{C} . \mathrm{C}_{00} \mathrm{H}_{48} \mathrm{Ge}_{2} \mathrm{~N}_{4} \mathrm{Si}_{2} \mathrm{Te}_{2}$ (Molmasse: 801,18); C 30,56 (ber. 29,88), H 6,04 (6,04), N 7,12 (6,99), 
Tc 33,58 (31,85)\%. 'H-NMR: $0,26(\mathrm{~s}, 12 \mathrm{H}) \mathrm{Si}-\mathrm{Me}, 1,58(\mathrm{~s}$, $36 \mathrm{H}) \mathrm{NBu}^{1}{ }^{19} \mathrm{C}-\mathrm{NMR}: 7,21\left(\mathrm{Si}-\mathrm{CH}_{3}\right), 33,66\left(\mathrm{C}-\mathrm{CH}_{3}\right), 53,95$ (C- $\left.\mathrm{CH}_{3}\right) ;{ }^{15} \mathrm{~N}-\mathrm{NMR}:-297,7 \mathrm{ppm} ;{ }^{29} \mathrm{Si}-\mathrm{NMR}: 4,50 \mathrm{ppm} ;{ }^{125} \mathrm{Tc}-$ NMR: 464,45 ppm ; MS: m/e (rel. Intensität) 801 (3,37), $\mathbf{M}^{\prime}$.

\section{$\left[\mathrm{Me}_{2} \mathrm{Si}(\mathrm{NtBu})_{2} \mathrm{SnSe}_{2}(9)\right.$}

$240 \mathrm{mg} \quad(3,04 \mathrm{mmol})$ schwart-granes Selenpulver werden in $50 \mathrm{ml}$ Toluol suspendiert. Dazu gibt man $5 \mathrm{ml}$ ciner $0,77 \mathrm{M}$ Lösung des Stannylens 2 in Toluol. Beim Erhitzen der grünschwarzen Suspension auf $80^{\circ} \mathrm{C}$ löst sich das Selenpulver langsam auf und es bildet sich eine zitronengelbe Lösung. Man filtriert die Lösung heiß, um sie von überschüssigem Selenpulver zu trennen und läßt sie dann langsam $(48 \mathrm{~h})$ in Ölbad bei $50-30^{\circ} \mathrm{C}$ abkühlen. Auf diese Weise crhält man $1,15 \mathrm{~g}(95,4 \%)$ gelber Nadeln. Diese sind sehr schlecht löslich. Schmp.: Zersetzung oberhalb $300^{\circ} \mathrm{C}$. $\mathrm{C}_{20} \mathrm{H}_{48} \mathrm{~N}_{4} \mathrm{Se}_{2} \mathrm{Si}_{2} \mathrm{Sn}_{2}$ (Molmasse: 795,6); C 30,33 (ber. 30,17), H 6,04 (6,08), N 7,06 (7,03)\%; ' $\mathrm{H}-\mathrm{NMR}$ : $0,31(\mathrm{~s}, 12 \mathrm{H}) ; 1,41(\mathrm{~s}, 36 \mathrm{H}) ;{ }^{13} \mathrm{C}-\mathrm{NMR}: 6,9\left(\mathrm{Si}-\mathrm{CH}_{3}\right), 35,5$ $\left(\mathrm{C}-\mathrm{CH}_{3}\right), 53,6\left(\underline{\mathrm{C}}-\mathrm{CH}_{3}\right)$; MS (CI): Intensität in Klammern: $799(8,2), 798(7,3), 797(9,6), 796(7,8)=M^{+}$.

\section{$\left[\mathrm{Me}_{2} \mathrm{Si}(\mathrm{NtBu})_{2} \mathrm{SnTe}_{2}(\mathbf{1 0})\right.$}

$0,175 \mathrm{~g}(1,40 \mathrm{mmol})$ schwarzes Tellur und $1,50 \mathrm{mmol}$ des Stannylens 2 werden in $40 \mathrm{ml}$ Toluol bei $80^{\circ} \mathrm{C}$ zur Reaktion gebracht. Es tritt eine langsame Rotfärbung der Losung ein. Nach $25 \mathrm{~h}$ wird das Reaktionsgemisch durch Filtrieren bei $60^{\circ} \mathrm{C}$ von metallischem Tellur befreit. Aus der orange-roten Lösung fallen beim langsamen Abkühlen auf $30^{\circ} \mathrm{C} 0,450 \mathrm{~g}(72 \%)$ roter Stäbchen an. Die Verbindung rersetzt sich an der Luft unter Schwarzfärbung; sie ist in allen gängigen organischen Lösungsmitteln sehr schlecht löslich. Schmp.: Zersetzung oberhalb $300^{\circ} \mathrm{C} . \mathrm{C}_{20} \mathrm{H}_{48} \mathrm{~N}_{4} \mathrm{Si}_{2} \mathrm{Sn}_{2} \mathrm{Te}_{2}$ (Molmasse: 893,4); C 27,34 (ber. 26,89), H 5,41 (5,47), N 6,27 (6,14)\%. 'H-NMR: 0,31 (s, $12 \mathrm{H})$, $1,46(\mathrm{~s}, 36 \mathrm{H}) ;{ }^{13} \mathrm{C}-\mathrm{NMR}: 7,8\left(\mathrm{Si}-\mathrm{CH}_{3}\right), 35,2\left(\mathrm{C}-\mathrm{CH}_{3}\right), 54,1$ (C- $\left.-\mathrm{CH}_{3}\right)$; MS (CI) (Intensität in Klammern): $886(0,02)$, $887(0,03), \quad 888(0,03), \quad 889(0,05), \quad 890(0,07) 891(0,06), 892$ $(0,08), 893(0,10), 894(0,08), 895(0,05), 896(0,07)=\mathrm{M}^{+}$.

\section{Literatur}

[1] D. H. Harris, M. F. Lappert, J. Chem. Soc., Chem. Comm. 1974, 895

[2] P. J. Davidson, D. H. Harris, M. F. Lappert, J. Chem. Soc., Dalton. Trans. 1976, 2268

[3] M. J. S. Gynane, D. H. Harris, M. F, Lappert, Ph. P. Power, P. Rivière, M. Rivière-Baudet, I. Chem. Soc., Dalton. Trans 1977, 2004

[4] M. Veith, M. Grosser, Z. Naturforsch. 37 B (1982) 1375

[5] A. Meller, C-P. Gräbe, Chem. Bcr. 118 (1985) 2020

[6] P. Jutzi, A. Becker, H. G. Stammler, B. Neumann, Organometallics 10 (1991) 1647

[7] W. A.Herrmann, M. Denk, J. Behm, W. Scherer, F. R. Klingan, H. Bock, B. Salouki, M. Wagner, Angew.
Chem. 104 (1992) 1489; Angew. Chem. Int. Ed. Engl. 31 (1992) 1485

[8] P. J. Davidson, M. F Lappert, J. Chem. Soc., Chem. Comm. 1973, 317

[9] M. Veith, Angew. Chem. 87 (1975) 287; Angew, Chem. Int. Ed. Engl. 14 (1975) 263

[10] W. P. Neumann, Chem. Rev. 91 (1991) 311

[11] R. W. Chorley, P. B. Hitchcock, M. F. Lappert, J. Chem, Soc., Chem. Comm. 10 (1992) 525

[12] M. A. Edelman, P. B. Hilchcock, M. F. Lappert, J. Chem. Soc., Chem. Comm 8 (1990) 1116

[13] M. Veith, O. Recktenwald, Z. anorg. allg. Chem. 459 (1979) 208

[14] D. Ellis, P. B. Hitchcock, M. F. Lappert, J. Chem. Soc., Dalton. Trans 1992, 3397

[15] S. Masamune, S. A. Batcheller, J. Park, W. M. Davis, J. Am. Chem. Soc. 111 (1989) 1888

[16] P. B. Hitchcock, H. A. Jasim, M. F. Lappert, W. P. Leung, A. K. Rai, R. E. Taylor, Polyhedron 10 (1991) 1203

[17] $M$. Veith, O. Recktenwald, E. Humpfer, Z. Naturforsch. 33 b (1978) 14

[18] H. Puff, R. Gattermayer, R. Hundl, R. Zimmer, Angew. Chem. 89 (1977) 556; Angew. Chem. Int. Ed. Engl. 16 (1977) 547

[19] A. Blecher, M. Dräger, Angew. Chem. 91 (1979) 740; Angew. Chem. Int. Ed. Engl. 18 (1979) 677

[20] M. Veith, S. Becker, V. Huch, Angew. Chem. 10] (1989) 1287; Angew. Chem. Int. Ed. Engl. 28 (1989) 1237

[21] Weitere Informationen zu den Kristallstrukturuntersuchungen sind beim Fachinformationszentrum Karlsruhe, Gesellschaft für wissenschaftlich-technische Information mbh, D-76344 Eggenstein-Leopoldshafen hinterlegt und können dort unter Angabe der Hinterlegungsnummer CSD-58245, der Autoren und des Zeitschriftenzitates erfragt werden.

[22] G. M. Sheldrick, Crystallographic Computing; G. M. Sheldrick, C. Kruger, R. Goddard (Herausgeber); Oxford University Press; London Vol. 3 (1985) 175

[23] G. M. Sheldrick: The SHELX Program, Computing in Crystallography; H.Schenk, R. Ollof-Hazenkamp, H. van Koningsveld, G. C. Bassi (Herausgeber); Delft University Press, Delft, Niederlande 1978, S. 34

[24] SHELXTL-PIUS (G. M. Sheldrick, A Program for Crystal Structure Determination, Version 4.0 1989, Siemens Analytical X-Ray Instruments, Madison WI)

[25] SCHAKAL 88B/V16: E. Keller, Kristallographisches Institut der Universität, Hebelstraße 25, D-79098 Freiburg i. Br.

Anschr. d. Verf:

Prof. Dr. M. Veith

Institut für Anorganische Chemie

der Universität des Saarlandes

Am Stadtwald

D-66123 Saarbrücken 